\title{
Le motif des mains croisées : Michèle Desbordes
}

\author{
Natacha ALLET \\ Université de Genève
}

\begin{abstract}
Dans La Demande, Michèle Desbordes remet en circulation une mémoire collective dont elle explore les failles, creuse les non-dits. Elle figure la rencontre entre un personnage historique, le peintre illustre Léonard de Vinci et un autre fictif, une simple servante. Cet article interroge la valeur d'un motif construit au fil des pages, celui des mains croisées, motif qui entre dans un lointain jeu d'écho avec un détail de La Joconde. Il appréhende la façon singulière, cryptée dont la fiction réinvestit ce motif pictural. Il examine d'une part le dispositif dans lequel il s'inscrit, qui relate l'histoire du geste, franchissant et refranchissant les frontières de l'art et de la vie, de l'intime et du partageable. Il tâche de montrer d'autre part que le motif tel que le réélabore La Demande mime, à la façon d'un emblème, son travail de mémoire.
\end{abstract}

Keywords : Desbordes, motif, mains croisées, Léonard de Vinci, anonyme

Encore pleine du silence dont elle s'arrache, l'écriture de Michèle Desbordes remet en circulation une mémoire collective dont elle explore les failles, creuse les non-dits. La Demande (1998) appartient à ce "genre » appelé la fiction biographique ou critique, qui revisite diversement la tradition ancienne des Vies. Lauteure figure la rencontre entre deux personnages, l'un historique, le génie universel, le peintre illustre Léonard de Vinci, et l'autre fictif, une servante qui lui aurait été attachée. Si le prénom de Tassine apparaît à l'orée du récit, et une seule fois, Vinci quant à lui n'est jamais nommé. Des indices permettent toutefois de l'identifier, qui ont trait à son œuvre ou à sa légende, et de situer l'histoire, malgré l'absence de date, durant les trois dernières années de sa vie (15I6-15I9), celles de l'exil en France sur l'invitation du roi. Desbordes raconte la progression de la relation qui se noue entre le vieux maître et sa servante paysanne qu'a priori tout sépare, elle raconte les habitudes qu'imperceptiblement ils prennent, leurs échanges muets, et nous conduit dans une tension croissante jusqu'à la demande que la servante adresse au peintre. Ranimant les traces d'un héritage culturel avec une discrétion toute particulière, elle s'attache au temps qu'il fait et aux jours qui passent, à la routine, accueille sans ironie le tout-venant de l'existence.

La parole étant à la fois banale et rare, dans La Demande, une attention spéciale est prêtée au jeu des regards, en accord avec l'inspiration picturale du texte, ainsi qu'aux gestes, ceux de la servante essentiellement, placée bien souvent sous l'œil du peintre. Aussi interrogerai-je de manière privilé- 
giée la valeur d'un motif, celui des mains croisées, que la fiction construit au fil des pages. Parlant dans un entretien de la genèse de son texte, Desbordes affirme qu'à l'origine de son livre, il y a l'image d'une « vieille assise près de la fenêtre, les mains croisées dans le creux de ses jupes » et que cette image fondatrice vient de sa mémoire intime : « elle porte[rait] la marque d'une de [s] es grands-mères ${ }^{1}$. Mais ce motif entre aussi dans un lointain jeu d'écho avec un détail de l'œuvre picturale la plus fameuse qui soit, devenue telle grâce aux Vies de Vasari et la relance du mythe au temps du romantisme, La Joconde, cette icône de l'art. On peut dire qu'il participe à ce titre d'une pensée du lieu commun, ne serait-ce qu'en raison de la postérité du tableau, imité, recyclé de manière plus ou moins irrévérencieuse de Raphaël à Duchamp, et au-delà. Il reste que la référence est cryptée, elle n'est pas désignée par Desbordes qui semble avancer dans son récit, comme son personnage, «à petits pas dans les futaines grises $»^{2}$. Je voudrais appréhender ici la façon singulière dont le motif pictural est réinvesti par la fiction critique. J'examinerai d'abord le dispositif dans lequel il s'inscrit, qui relate l'histoire du geste, franchissant et refranchissant les frontières de l'art et de la vie, de l'intime et du partageable. Je montrerai ensuite que le motif tel que le réélabore l'œuvre littéraire mime, à la façon d'un emblème, le travail de mémoire qu'elle engage.

\section{La circulation du motif commun}

On connaît certes mieux le sourire de La Joconde que le détail de ses mains croisées. Mais Léonard a étudié avec intensité l'anatomie de la main dans ses années tardives, comme en témoigne un ouvrage publié par deux médecins, La Main de Léonard de Vincỉ. Et La Demande semble s'en souvenir, en évoquant parmi les dernières visions du maître italien: « Des mains et des mains » (I4O). Le motif pictural émerge selon toute vraisemblance avec $\mathrm{La} \mathrm{Jo}$ conde ${ }^{4}$. La pose de ses mains qu'« il a fallu plus de vingt-cinq ans à Léonard pour mettre au point», suivant Daniel Arasse, a été «abondamment reprise,

\footnotetext{
I Entretien avec Jacques Le Scanff publié dans la revue Le Préau des collines, 5, «À propos de Michèle Desbordes. Éléments d'une biographie (du rêve) ", Paris, 2002, pp. 3I-32.

2 Michèle Desbordes, La Demande, Gallimard, "Folio », 200I, p. 24. Dorénavant, les références à cette édition seront placées entre parenthèses dans le texte.

3 Dominique Le Nen et Jacky Laulan, La Main de Léonard de Vinci, Paris, Springer, 2oro. Les auteurs de cet ouvrage étonnant ont réalisé de véritables dissections à partir des dessins de Léonard et livrent la trace photographique de leur travail de reconstruction historique.

4 Jan Blanc, qui choisit de reproduire ce motif sur la couverture d'un ouvrage consacré au peintre, ne lui trouve nul antécédent ; il signale seulement le Portrait de Ginevra de' Benci (I475I478) dont " on peut penser que les mains du modèle étaient visibles dans l'état originel de l'œuvre, selon une composition proche de celle de La Joconde " (Ioo \% Léonard de Vinci, Paris, Éditions de La Martinière, 20II, pp. 38 et I49).
} 
par Raphaël en particulier $»^{5}$. Son succès « a pu tenir au fait », selon l'historien d'art, qu'elle correspondait, « dans la société contemporaine, à celle que devaient adopter en public les jeunes femmes ${ }^{6}$. Mais sa valeur ne se limite pas aux «implications sociales d'une gestuelle codée ${ }^{7}$. Les mains croisées de Mona Lisa sont indissociables, à ses dires, de l'effet du tableau « qui vient imaginairement vers son destinataire ", tout en " maintenant une distance infinie ${ }^{8}$, selon un double mouvement d'attraction et de retrait ${ }^{9}$. Je préciserai encore que le motif pictural relève du topos par-delà cette codification oubliée, dans ma perspective, du simple fait qu'il appartient à La Joconde, « le tableau le plus célèbre du monde ${ }^{\mathrm{IO}}$, et fait signe en sa direction.

De ce motif commun, éculé, la fiction littéraire fait un geste singulier et vital, celui de la servante que guette le peintre, un geste quotidien qui résume Tassine. Tout porte à croire que La Demande reverse le motif dans le contexte dont il a été extrait pour être hissé sur la fameuse toile. Parmi d'autres indices de ce travail de défixation, de transbordement en-deçà de l'œuvre, mentionnons cette allusion, cet espèce de clin d'œil : «Quand elle [Tassine] tardait à sourire, il [le maître] se demandait pourquoi » (II3). Desbordes semble bien restituer l'univers sensible d'où provient le portrait, d'autant qu'elle évoque la relation de l'artiste avec son modèle. S'habituant à son regard sur elle, la servante se dit en effet que le maître peut l'observer " comme il observ[e] un arbre ou une couleur de ciel » (54). Pourquoi ne pas situer le récit à Florence dès lors, où la Joconde a été peinte, plutôt qu'en France qui forme le dernier séjour de Vinci ? Sur les bords de la Loire, le vieillard se laisse " bercer » par « l'habitude, la femme » à ses côtés qui « enfoui[t] » sa peine «sous la patiente répétition des gestes de tous les jours », il se laisse porter par la routine : « le soleil se levait et se couchait, le ciel était bleu, le ciel était gris » (IIO). La vie monotone et rurale que retrace La Demande renvoie à un univers postérieur à la réalisation de La Joconde, mais qui plonge en réalité dans une mémoire intime, liée à la création :

[...] il finissait par se dire qu'elle était là depuis toujours, parfois même c'était comme s'il se souvenait d'elle qui l'emportait dans chacun de ses pas et la grande allongée de ses jupes, il pensait à l'enfance dans les collines, se souvenait de longues jupes [...] (III).

5 Daniel Arasse, Léonard de Vinci : le rythme du monde, Paris, Hazan, 1997, p. 402.

6 Ibid., p. 405.

7 Ibid., p. 406.

8 Ibid., p. 402

9 Nous verrons que ce double mouvement trouve un écho dans l'attitude de Tassine, entre retenue et effraction.

IO Ibid., p. 386 . 
C'est le souvenir de son enfance et de sa mère paysanne, " la vieille qui pendant vingt ans l'avait attendu dans les collines du côté d'Empoli » (58), qui remonte en lui au contact de Tassine. S'amorce un mouvement d'anamnèse où les deux femmes tendent à se confondre. À la faveur de cette association, la fiction retrempe le motif pictural des mains croisées dans la simplicité d'un quotidien sans éclat.

La vie ordinaire et le savoir populaire, intuitif qui lui est lié, forment un contraste dans La Demande avec le monde majeur de l'art et de la culture livresque, avec la « gloire et la beauté » qu'on ne demande « jamais qu'aux Italiens » (32 et 34). La reprise littéraire de ce motif des mains croisées signale - souterrainement - la tension entre ces univers opposés que Desbordes met en présence et cherche dans son récit à croiser. Elle signale aussi la circulation qu'elle vise à opérer. Elle est une manière, discrète, mais emblématique, de faire le lien, de jeter un pont entre des pôles divergents : à Vinci les carnets, à Tassine les seaux ; à l'une la parole banale («Elle continuait à dire des nouvelles de la ville [...] », 83), à l'autre les grands sujets (« Eux parlaient de l'Italie et des guerres qui n'en finissaient pas [...] ", 84); à l'un les voyages, à l'autre l'espace domestique. Mais tout le récit travaille à réduire l'écart, à atténuer les différences de valeurs entre les mondes qu'il entrelace : l'artiste exilé est lui-même au service du roi, ses fresques s'abîment, son univers se rétrécit, on le voit tenir ses comptes et il semble apprendre de Tassine, de son endurance. Quant à la demande que fait la servante, elle est exemplaire : ayant vu des dessins anatomiques du peintre, elle lui offre son corps, à sa mort, comme matériau, au profit de l'œuvre et du savoir, dans une sorte d'accomplissement de sa fonction ; le sacrifice du corps réunit ainsi les deux mondes, prosaique et inspiré. Le style mime cet effort de déhiérarchisation, par la pratique massive de l'asyndète, de l'énumération, et par le lyrisme qu'induit le rythme, le retour du même, voire la ponctuation ; la parole la plus triviale prend des allures de vers, se poétise : «Elle disait qu'elle avait mal dormi, la poule avait chanté le coq, ou la chevêche crié jusqu'au matin » (83).

Or Desbordes montre aussi comment le geste de Tassine se fige, sous le regard du peintre, et devient archétypique, prêt à retraverser les cloisons perméables de l'art et de la vie :

Plus que jamais elle se taisait, [...] en elle parlaient toutes celles qui s'asseyaient sans rien dire près des fenêtres et croisaient les mains dans leurs jupes, comme en lui [son fils] qu'elle regardait d'un regard fatigué vivaient tous les idiots [...]. Comme elle qu'il [le maître] regardait était la servante, toutes les servantes depuis que le monde existait, depuis que le temps n'en finissait pas [...] (IO2-IO3, je souligne).

La fiction restitue ainsi la pose de la servante à une universalité, non 
seulement artistique, mais aussi vitale : elle la rend à une communauté. À ce motif emprunté à une mémoire culturelle usée, elle confère un contenu en même temps qu'une existence renouvelée, litéraire.

\section{Du nom au motif}

«Il savait d'elle qu'elle s'appelait Tassine »; ainsi s'ouvre La Demande qui ne délivre pas le nom du maître venu d'Italie, celui que précède sa réputation et dont l'arrivée au manoir est attendue, mais le simple prénom, inventé, de celle qu'on découvre peu à peu, de l'anonyme.

En passant sous silence le nom de l'artiste glorieux, la fiction de Desbordes crée autour de lui une zone d'indétermination et tient en suspens tout un horizon référentiel : au goût du vrai, du réel tangible, historique, elle privilégie celui du possible, sa force heuristique, comme le laisse déjà présager le sous-titre « histoire ». Il n'empêche qu'elle puise à la source d'un savoir constitué et précis. On lit d'ailleurs sur la quatrième de couverture que le personnage du maître est « un peu Michel-Ange, beaucoup Léonard de Vinci ». Et Desbordes lève toute espèce d'hésitation dans ses entretiens, en nommant explicitement Léonard ${ }^{\mathrm{II}}$. Son récit s'inspire de fait de la légende du peintre, façonnée par Vasari ${ }^{12}$ et diversement reprise et amplifiée, réinterprétée : il recycle des données connues de la biographie de Vinci, comme sa traversée des Alpes, les trois tableaux (dont fait partie La Joconde) emportés en France, son dernier séjour auprès de François I $^{\text {er }}$, l'organisation de fêtes ; il exploite le souvenir, crucial dans la contribution de Freud ${ }^{13}$ au mythe, de la mère paysanne, qui vient s'associer sous la plume de Desbordes, comme on l'a vu, à la figure de Tassine; il fait allusion aux carnets de l'artiste et à ses fresques, à l'inachèvement de son œuvre ${ }^{\mathrm{I} 4}$; il mentionne enfin l'élève Salaï

II Dans l'un d'eux, par exemple, elle affirme que Léonard de Vinci est un hasard : « [...] un jour que j'étais à Chambord, au pied de ce grand escalier que vous connaissez, et qui a sans doute été conçu d'après les dessins de Léonard (escalier fascinant car le principe en est que les gens qui montent ne voient pas ceux qui descendent), j'ai commencé à penser très fort à Léonard. [...]. Et le livre était là [...].» (Le Préau des collines, op. cit., p. 33).

I2 Giorgio Vasari, «Vie de Léonard de Vinci. Peintre et sculpteur florentin », in Les Vies des meilleurs peintres, sculpteurs et architectes, trad. et éd. André Chastel, t. 5, Paris, Berger-Levrault, 1983.

I3 Dans Un souvenir d'enfance de Léonard de Vinci, Sigmund Freud insiste sur la naissance illégitime de l'artiste et son attachement à sa mère dont la réminiscence serait devenue obsessionnelle au temps de La Joconde : « Le sourire de Mona Lisa del Giocondo réveilla, en l'homme fait qu'était alors Léonard, le souvenir de la mère de ses premières années. Et depuis lors, chez les peintres de l'Italie, les madones et les nobles dames eurent l'humble inclinaison de tête et le sourire étrange et bienheureux de la pauvre paysanne Caterina, qui avait donné au monde ce fils de gloire, destiné à l'art, à l'investigation, à la souffrance » (trad. Marie Bonaparte, Paris, Gallimard, 1977, p. 108).

I4 Sa difficulté à finir, l'inachèvement de ses toiles et de nombre de ses projets forment un 
et rêve autour de « l'Adoration » $(56)^{15}$ ou de « l'ange du Baptême, la première œuvre » $(62)^{16}$. Revisitant ainsi la matière de sa vie, Desbordes s'intéresse moins à Léonard toutefois qu'à la servante qu'on lui donne et à leur rencontre. Si elle retient ce qu'il incarne, le génie ou la grâce, l'immense savoir, elle choisit de le figurer, plutôt qu'au sommet de sa gloire, à l'approche de la mort, au moment où sa main tremble et sa mémoire vacille - et travaille diversement à égaliser les destins.

L'effacement du nom chargé, surdéterminé de Vinci prend à cet égard un sens supplémentaire. Il permet de s'affranchir de la référence, mais aussi de mettre en sourdine la renommée - le génie florentin étant l'archétype de la figure mémorable - et de prêter l'oreille aux voix oubliées. Sans doute faut-il cet amuïssement pour ne pas les recouvrir, les étouffer. Il est remarquable que l'émergence de Tassine dans l'univers de la fiction décrive un mouvement d'arrachement à une mémoire collective et orale, infiniment fragile, minuscule :

Une paysanne, c'est ce qu'on leur avait dit, et venue des tourbières, plus bas après la première forêt, de celles qui servaient dans les maisons du fleuve, avaient toujours servi [...]. Elle était petite et frêle, comme une enfant se dirent-ils [le maître et les élèves], le visage était fin [...] (28, je souligne).

La voix narrative se greffe ici sur la rumeur, l'on-dit, qu'elle reprend et prolonge, par le relais des peintres. Renouant avec une mémoire perdue, locale et éphémère, elle tire de l'ombre le personnage infime de la servante et l'isole, le détache du groupe social. Elle donne à celle que nul ne regarde, dissimulée sous sa coiffe et la toile de ses jupes, la chance d'apparaître :

C'est à peine s'ils la virent en entrant, elle lavait dans les cours, allait et venait le long des murs avec les seaux et les linges mouillés, tordus d'un coup de poignet, petite, tout habillée de gris, seul brillait le blanc de la coiffe [...] (23).

Appréhendée d'abord de l'extérieur, réduite à sa coiffe, à ses tâches, Tassine se dévoile peu à peu ${ }^{17}$. Elle s'individualise à mesure que la relation s'ap-

motif récurrent de sa légende, dont parle Vasari et que rappelle Daniel Arasse dans L'Univers de Léonard de Vinci, en un chapitre intitulé « L'Universel inachevé » (Paris, Henri Scrépel, 1978, pp. II-25).

I5 C'est ici une allusion à L'Adoration des mages de Vinci.

I6 Vasari déjà mentionne l'ange célèbre peint par Vinci sur la toile de son maître Verrocchio, Le Baptême du Christ («Vie de Léonard de Vinci. Peintre et sculpteur florentin », op. cit., p. 35).

I7 Il vaut la peine de noter que la coiffe de la servante, dont n'émerge d'abord qu'un « léger friselis, une mèche lissée sur la tempe » (28) laisse apparaître pour finir « deux nattes claires entourant ses joues » (II6). Sa description scande ainsi les étapes de la relation, les suggère 
profondit : on accède par fragments à sa vie intérieure, grâce au maître qui l'observe et l'imagine, interprétant ses actions et ses gestes, les réévaluant, jusqu'à l'événement décisif de sa prise de parole, longtemps différée. La fiction arrache donc à l'oubli le personnage improbable de l'anonyme, le fait exister. Elle le nomme et laisse émerger sa voix, accueille sa parole dans l'espace littéraire ; elle travaille à rétablir un équilibre.

Le motif des mains croisées, tel que le reprend et le met en place $\mathrm{La} \mathrm{De-}$ mande, participe à mon sens d'une opération analogue. Il est tacitement emprunté à la peinture, la référence à La Joconde subissant la même éclipse que le nom de Léonard. Son insistance dans le récit prépare l'événement de parole qui en est l'acmé, par touches réitérées : assise près des fenêtres, la servante « regard[e] ses mains dans le creux de ses jupes » $(44 \text {, je souligne })^{18}$, puis les «croise et les décroise » (52 et 87 , je souligne), à diverses reprises, avant de se tenir immobile, les mains croisées, sous l'œil des peintres (IOI-IO5). Leur attention croissante à ce geste cherche à comprendre, et intensifie le silence que brise l'« incroyable demande » (I25) - là où le silence est explicitement associé à la servitude, à l'oppression sociale et la parole au pouvoir. À leurs yeux, la posture de Tassine dit « l'habitude et la résignation » (IO2), la retenue, elle exprime la souffrance rentrée, la honte, et délivre toute son histoire. Le détail réapparaît une dernière fois après que la servante a formulé sa requête, pour devenir le signe qui la caractérise, l'identifie : « Elle croisait les mains sur ses genoux, il [le maître] la retrouvait, la reconnaissait » (I25). À son tour, et à un autre niveau, la voix narrative fait de ce motif l'attribut de Tassine : dans l'imaginaire du lecteur, elle la figure les mains croisées. Elle lui assigne ainsi le geste de Lisa del Giocondo, en une étrange surimpression qui met sur le même plan des personnages socialement opposés - la servante anonyme et la riche épousée, immortalisée par l'art, infiniment célèbre - voire les substitue l'un à l'autre.

Ce subtil jeu d'échange permet de mesurer la nature particulière du rapport que la fiction de Desbordes entretient avec la mémoire culturelle, relation qui s'éclaire à la lumière de l'histoire relatée, par une forme d'homologie. Sur le plan de la diégèse, la demande de la servante - qui est un don, celui de sa dépouille - est vécue comme une « indiscrétion » (I20), une « outrecuidance » (I22), un effort sanctionné par des larmes. Elle consiste de fait en une petite effraction. En faisant entendre sa subjectivité, sa voix, la servante rompt avec le geste qui lui est propre et la réserve qu'il signale, elle sort de son rôle; et le vœu qu'elle énonce, alors qu'elle s'identifie aux « morts des hospices » qu'elle a vus disséqués dans les anatomies du maître, «ignorés de tous » et privés de sépulture, « soumis pour la dernière fois » (I26-I27), in-

sans les nommer.

I8 Voir aussi p. 70. 
duit une prise de conscience, en désignant la violence de la condition qu'elle partage avec eux. Cette effraction pourtant respectueuse, il semble que la fiction la rejoue, à sa manière. Elle ne rompt pas en effet avec le patrimoine culturel qu'elle tient à distance, mais voit en lui une médiation, y cherche des ressources, atténuant le fracas de la notoriété pour faire entendre ce qu'il dissimule. Le souhait de Tassine qui offre son corps au peintre, à sa mort, est de servir encore et d'obtenir aussi, peut-on imaginer, un salut par l'art. Or La Demande accède à son désir secret et fait le portrait qui n'a pas eu lieu, sans pour autant obéir à une logique subversive. Malgré sa part critique en effet, elle n'offense pas les modèles, n'« en fini[t pas] avec les chefsd'œuvre $»^{19}$. Si Desbordes figure l'anonyme, à la manière du peintre flamand Jan Van Eyck en I $432^{20}$, elle conserve dans son récit l'ombre de Vinci et celle de La Joconde; elle épouse qui plus est le regard du maître. Elle ménage donc une tension entre les personnages et les mondes qu'elle invite à se rejoindre, dans un souci d'inclusion. Elle vise à reconfigurer le mémorable - et le motif des mains croisées sous sa plume peut être considéré comme l'emblème de cette ambition.

Quel intérêt trouve-t-elle enfin à effectuer un tel emprunt à l'art plutôt qu'à la littérature ? La peinture préserve des formes et des gestes, des attitudes perdues - qui sont les témoins muets de mondes défunts. Elle détient une réserve de traces, comme en souffrance. Les mains croisées de Tassine, dans La Demande, exhibent leur retenue, elles s'offrent à la lecture, la réclament :

Le soir elle restait plus longtemps assise près des fenêtres à se taire et regarder au-dehors, croiser et décroiser les mains dans le creux de ses jupes. $I l$ se disait que chaque soir depuis qu'elle servait dans les maisons du fleuve elle s'était assise près des fenêtres, avait croisé et décroisé les mains dans le creux de ses jupes, avec ces choses à dire qu'elle ne disait pas et ne dirait jamais, les taisant et montrant qu'elle les taisait dans des silences amers, comme si les taire et montrer qu'elle les taisait appartenait à la tristesse et à la solitude, au gris infini [...] (87, je souligne).

Observant la servante qui croise les mains en silence près des fenêtres, le peintre finit par entendre la "voix muette, cassée, la plainte silencieuse " et par « comprendre» (I04-IO5). À ce titre, son attention active, herméneu-

I9 Il s'agit là d'une expression d'Antonin Artaud, d'un titre qu'il donne à l'un des essais du Théâtre et son double (in Euvres, éd. Evelyne Grossman, Paris, Gallimard, 2004, p. 549).

20 Hans Belting a commenté son célèbre tableau intitulé Timotheos (ou Leal Souvenir) où « le peintre, comme un notaire, témoigne de l'existence du jeune homme qui devait survivre grâce à son portrait "; voir " Mémoire et portrait. Face et interface du premier sujet "moderne" ", in Le Texte de l'oeuvre d'art : la description, éd. Roland Recht, Strasbourg, Presses universitaires de Strasbourg, 1998, p. 172. 
tique s'avère révélatrice du rôle que se donne ici la fiction. Dans le sillage du maître, La Demande entend prêter l'oreille à ce qui se tait, à ce qui n'a pas été jugé digne d'être exprimé, reçu ou conservé dans la mémoire collective ou officielle.

S'il s'agit de donner une voix aux oubliés de l'histoire, comme on l'a vu, il s'agit aussi de laisser affleurer les voix intimes, refoulées, de ceux qui y ont une place. Car même pour le peintre qui obéit à ses maîtres, vient en France en réponse à une commande royale, même pour le génie de la Renaissance, il y a de l'inexprimé : ce qu'il « avait à dire » il «ne le disai[t] pas », il « ne le dirai $[t]$ pas », il «ne le dirai [t] jamais » (34). Le geste des mains croisées de Tassine désigne cela aussi, l'inaccompli des vies où s'égalisent les destins.

La Demande se livre en somme à un vaste travail de reconfiguration mémorielle. À une mémoire culturelle affectivement désertée, mécanique, elle oppose celle, collective, de la vie quotidienne et routinière, paysanne, qu'elle réinvestit. Mais elle associe le patrimoine à son entreprise, et le lie secrètement à ce qu'il néglige ou opprime, laisse dans l'ombre - dans un geste de conciliation et de réparation. Elle introduit du jeu entre les personnages et les mondes qu'elle met en tension, entre l'art et la vie. Relançant le motif pictural des mains croisées de La Joconde, elle le déporte, le remotive et lui donne un contenu, le rend à une collectivité anonyme, celle des servantes. Elle raconte toute l'histoire du geste, en amont de sa fixation, dans la diégèse, et en aval, dans l'acte même de sa reprise littéraire. Et elle le fait parler, pour donner voix aux anonymes, laissant entendre qu'il les individualise. La reprise littéraire du motif pictural est aussi, pour La Joconde, un bain de jouvence. Elle invite en effet, tout en nuance et en dissimulation, à des relectures du tableau. Que disent les mains croisées de cette femme, l'épouse d'un commanditaire ? Et si ce geste était celui aussi du peintre, en mal d'expression, voire de l'écrivaine ? La Demande ne peint pas la servante en Joconde, elle peint l'envers de La Joconde. 